\title{
Characterizing Atomic Ordering of High Entropy Alloys Using Super-X EDS Characterization
}

\author{
Robert E. A. Williams ${ }^{1,2}$, Brian Welk ${ }^{1,2}$, Jake Jensen ${ }^{1,2}$, Bryan D. Esser ${ }^{1}$, David W. McComb ${ }^{1}$, Hamish L. \\ Fraser $^{1,2}$ \\ 1. Department of Materials Science and Engineering, The Ohio State University, 477 Watts Hall, 2041 \\ College Road, Columbus, OH 43210, USA \\ 2. Center for Accelerated Maturation of Materials, 1305 Kinnear Road, Columbus, OH 43212, USA
}

High entropy alloys (HEA), more recently referred to as compositionally complex alloys (CCA), are a new group of alloys receiving a great deal of attention because of the potentially remarkable balance of properties they are expected to exhibit. They offer new pathways to lightweighting in structural applications, new alloys for intermediate and elevated temperature components, and new magnetic materials[1,2]. To realize their potential, however, requires considerable alloy development that will rely on application of integrated computational materials (science and) engineering (ICME), which requires accurate computational models predicting their performance in addition to a detailed knowledge of, for example, their deformation mechanisms. Often, these alloys consist of a mixture of ordered and disordered phases, and because of the compositional complexity, it is necessary to know the nature (i.e., degree of order, site occupancy, and presence of anti-site defects) in the ordered phases if effective models of the deformation behavior are to be developed[3]. These various metrics require accurate compositional measurements at the atomic scale.

The work to be presented involved determining the degree of order present in a model B2 compound consisting of Al- 25 at. $\% \mathrm{Ni}-25$ at. \%Co. In the past, ALCHEMI has been used to indicate the trend in sub-lattice occupancy but has not been able to provide determinations at to the actual degree of order[4]. Such determinations would require a direct characterization of the actual sub-lattice compositions. Recent technological improvements in STEM probe aberration correction and large collection angle, silicon drift detectors (SDD) for XEDS collection have provided unprecedented quality in XEDS spectral images. The incorporation of a DCOR, for probe correction up to the $5^{\text {th }}$ order, permits the use of larger probe forming apertures while reducing the probe diameter and the SDD have significantly improved collection efficiency over previous generation SiLi detectors. The benefits from this design are multifaceted in that the probe diameter is decreased and the probe current is increased, resulting in better spatial resolution and an increased analytical signal for detection by SDDs.

As technological advancements have improved the quality of the data collected, the physics of XEDS quantification has not yet evolved. Quantification of apparent atomic resolution XEDS suffers from the same difficulties as traditional XEDS quantification, moreover collection of apparent atomic resolution XEDS is performed on zone, an undesirable condition that maximizes electron channeling. Aside from the normal difficulties associated with XEDS quantification, the apparent atomic resolution XEDS data is complicated by column "cross-talk" from delocalization and contributions from phonon scattering [47].

The results shown in Fig $1(\mathrm{a}, \mathrm{b})$ were collected using a FEI Titan Themis ${ }^{\mathrm{TM}}$ 60-300 equipped with a DCOR probe corrector and Super-X EDS collection system with $\sim 200$ pA beam current and $25 \mu$ s dwell time/pixel. In Fig. 1 (a), the XEDS maps of the elemental distributions over the two sub-lattices of the 
B2 compound in the sample are shown, overlaying the HAADF image. Clearly, spatially-resolved spectral collection, at the atomic scale, has been achieved. The compositional profiles obtained are shown in Fig. 1 (b), from which it is clear that one sub-lattice is occupied mainly by Al atoms and the other sub-lattice consists mainly of $\mathrm{Ni}$ and $\mathrm{Co}$. As mentioned previously, quantification of this data is not trivial and a variety of physics-based and gaussians-based methods have been attempted to accurately extract chemical compositions from the XEDS spectral data. These results and methods will be discussed as well as suggestions for improvement.

\section{References:}

[1] B. Cantor, et al., Materials Science and Engineering: A 375 (2004): p. 213-218.

[2] J-W. Yeh, et al., Advanced Engineering Materials 6, no. 5 (2004): p. 299-303.

[3] J-W. Yeh, et al., Metall Mater Trans A 35 (2010): p. 2533.

[4] D. H. Hou, et al., Philosophical Magazine A, 74(3), (1996): p. 741-760.

[5] B. D. Forbes, et al., Physical Review B 86(2), (2012): 024108.

[6] N. R. Lugg, et al. Applied Physics Letters 101(18), (2012): 183112.

[7] L. J. Allen, and S. D. Findlay. Ultramicroscopy in press (2014).

[8] G. Kothleitner, et al., Physical Review Letters 112(8), (2014): 085501.
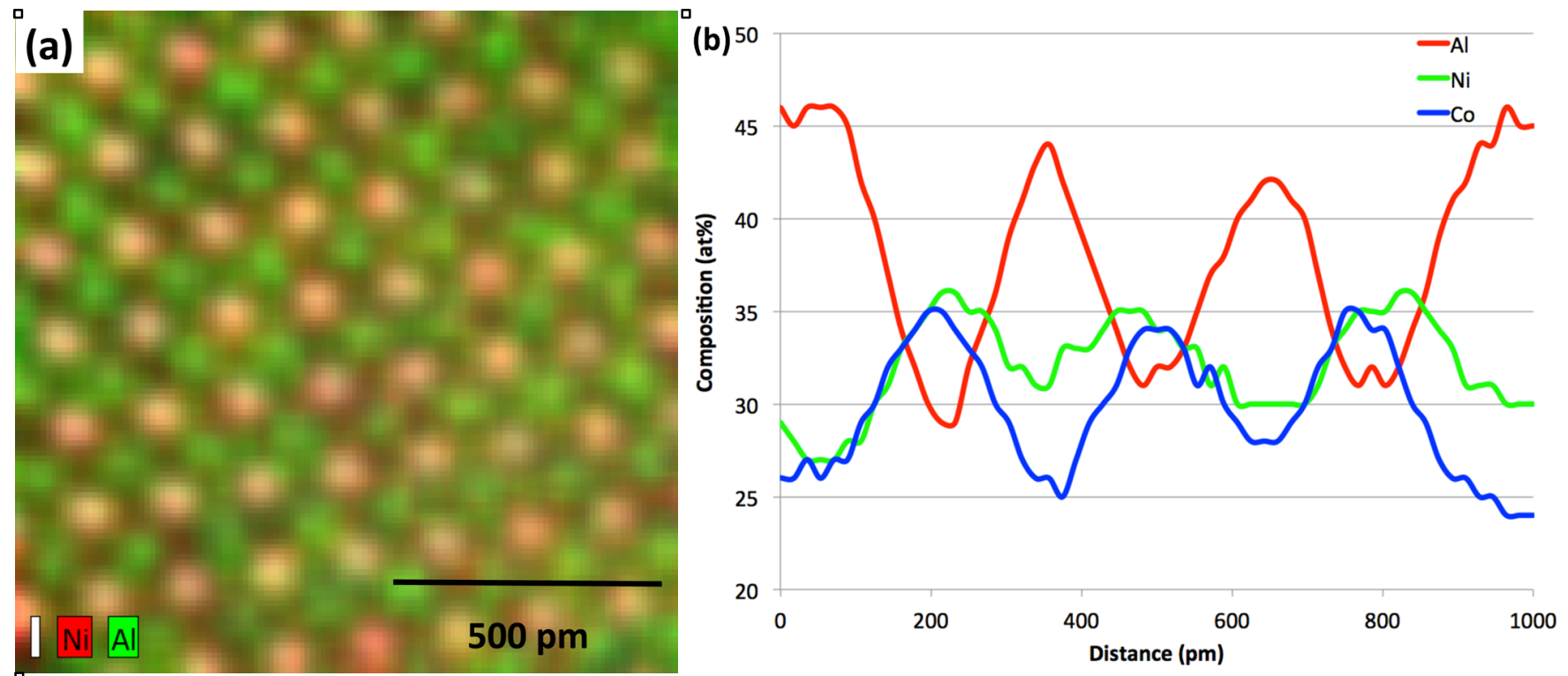

Fig. 1. a) XEDS map (overlaying a HAADF image) of the atomic columns in the model B2 compound with the beam parallel to $<100>$. b) XEDS compositional profiles plotted across a set of columns showing that one of the sublattices consists mainly of $\mathrm{Al}$, while the other mainly $\mathrm{Ni}$ and Co. 